\title{
Neuropsicología clínica
}

\section{Revisión y previsión \\ W. H. Buffery}

Universidad de Londres

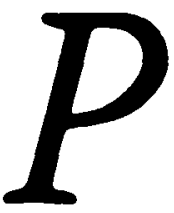

Podemos dar por descontado que, en la actualidad, cualquier teoría de la conducta será necesariamente inadeucada e incompleta. Pero por el hecho de que nosotros no hayamos encontrado aún cómo reducir la conducta al control del cerebro no podemos deducir que nadie vaya a poder bacerlo en el futuro."

De la Introducción a la Organización de la Conducta: Una teoría Neuropsicológica. De D. O. Hebb (1944).

\section{INTRODUCCION}

Es mi tesis que la neuropsicología clínica está a punto de alcanzar su madurez. El neuropsicólogo clínico no sólo puede hacer una contribución única a la comprensión de los problemas fundamentales de la relación entre la mente y el cerebro humano, sino que puede revolucionar el tratamiento de los pacientes que sufren diversas disfunciones del sistema nervioso superior, debido a las implicaciones terapéuticas de las disciplina. Vamos a presentar esta tesis en el contexto de una revisión del desarrollo y la contribución actual de la neuropsicología clínica y de una evaluación de su potencial.

\section{EL DESARROLLO DE LA NEUROPSICOLOGIA CLINICA}

En 1870 Fritsch e Hitzig criticaban la práctica que prevalecía en los exámenes neurológicos consistente en juzgar el nivel intelectual del paciente basándose en una exploración informal. Manifestaban la necesidad de contar con medidas estandarizadas y objetivas de las funciones mentales. Esta demanda tuvo una respuesta en 1905, cuando Binet y Simon pasaron pruebas formales a niños retrasados y con lesiones cerebrales en París; en 1907, cuando Bekhterev fundó el Instituto Neuropsicológico de Leningrado, y en 1909, en que Burt publicó sus resultados con pruebas experimentales de inteligencia en Londres. En 1934 Strauss y Savitsky podían escribir lo siguiente:

(*) De Rachman S. (Ed.) Contributions to Medical Psychology, vol. I. Oxford. PerGâmûn, 1977. 
«Ya han pasado los días en que el neuropsiquíatra puede hacer a la persona con lesión unas pocas preguntas y, como resultado de una breve entrevista, formarse una opinión sobre si están o no intactos todos los procesos psicológicos, la capacidad adaptativa de los pacientes y la constitución de su personalidad.»

En 1939 Rylander informaba de que los pacientes con tumores en el lóbulo frontal mostraban deficiencias intelectuales en ciertas pruebas psicológicas y que dichas diferencias no se observaban en un examen clínico más tradicional. En realidad, Rylander juzgaba que las evaluaciones psiquiátricas del estado mental son: «Una madeja enredada de observaciones objetivas y opiniones subjetivas.»

Y recomendaba su sustitución por los tests y las medidas más científicas y uniformes de la psicología, que permitirían una comparación significativa de los resultados obtenidos por distintos pacientes. Esta recomendación tiene su eco en muchos textos recientes de neurología (Birch, 1964; Burgermeister, 1962; De Jong, 1967; Gilbert, 1969; Small, 1973; Smith y Philippus, 1969; Weschler, 1963).

Desde la segunda guerra mundial han aumentado notablemente los laboratorios psicológicos de los hospitales, las facultades de Medicina, los centros de estudios neurológicos y de investigación, y se ha incrementado también la prácrica de realizar pruebas psicológicas formales a niños o adultos con alteraciones neurológicas sospechosas o confirmadas. Pero ¿cuáles son los frutos que va a dar esta siembra? ¿Cuál es, en realidad, la naturaleza de este árbol y de sus ramas?

\section{Neorpsicología clinica}

Davidson (1974) distingue tres áreas de investigación neuropsicológica: la neuropsicología experimental, la neurología conductual y la neuropsicología clínica. Las tres se ocupan de lo que Davidson denomina «relaciones entre cerebro y conducta», pero con diferentes objetivos y metodologías.

El neuropsicólogo experimental investiga los principios fundamenta: les de la función cerebral. Ocasionalmente pasa pruebas a seres humanos, pero con más frecuencia trabaja con animales en condiciones experimentales cuidadosamente controladas (ver las revisiones de Drewe, Ettlinger, Milner y Passingham, 1970). A pesar de las críticas recientes (Lockard, 1971) el carácter comparativo de gran parte de estas investigaciones no ha mitigado el entusiasmo de los neuropsicólogos experimentales ni su tendencia a hacer generalizaciones, muchas veces sin justificación, a través de la escala filogenética hasta llegar a la condición humana. Con todo, el estudio del cerebro animal tiene interés por sí mismo y algunos de los procedimientos de prueba han demostrado ser útiles en situaciones clínicas. Por ejemplo, Prisko (citado por Milner, 1964) investigó la memoria a corto plazo de pacientes epilépticos antes y después de una operación de cerebro, utilizando una versión modificada de la técnica de estímulos complejos desarrollada primero por Konorski (1959) para utilizarla con animales y adaptada después por Stepien y Sierpinski (1960) para los humanos. 
A diferencia de la neuropsicología experimental, el área de la neuropsicología a la que Meier (1964) denomina «neurología conductual» (ver también Pincus y Tucker, 1974) se centra en el paciente humano, pero a diferencia de la neuropsicología clínica acentúa más las definiones conceptuales de la conducta que las operacionales. El neurólogo conductual examina al paciente individual en profundidad y diseña pruebas para detectar cualquier desviación del funcionamiento «normal» en vez de medir cuantitativamente la conducta, situándola en dimensiones continuas, como hace el psicólogo clínico con sus pruebas psicométricas. Probablemente el mejor ejemplo de neurología conductual es el que nos proporciona el trabajo de Luria $(1962,1963,1968,1971)$, que trata de integrar los resultados de un examen imaginativo e idiosincrático de cada paciente (Christensen, 1975) con un sistema conceptual de neuropsicología humana (Luria, 1973), sistema comprehensivo aunque quizá un tanto prematuro. En cualquier caso, otros han demostrado que pueden realizarse estudios valiosos de casos individuales sin situarlos en el contexto de una teoría neuropsicológica elaborada (Oldfield y Williams, 1961; Warrington y Shallice, 1969; vid. también Buffery, 1974, p. 239).

La neuropsicología clínica se ocupa del diagnóstico, la evaluación y el pronóstico de pacientes con disfunción del sistema nervioso superior y está comenzando a contribuir a su tratamiento. El neuropsicólogo clínico mide la inteligencia, la habilidad senso-motora y la personalidad de pacientes con una lesión cerebral que se conoce o sospecha y las mide con tests que normalmente están estandarizados y sirviéndose de observaciones de la conducta, definida en términos operacionales y cuantificada en distribuciones continuas. Las puntuaciones de los pacientes se comparan con las de sujetos de control y el resultado se evalúa mediante análisis estadísticos. Además de diferenciar la lesión cerebral de otras posibilidadades diagnósticas, el neuropsicólogo clínico se preocupa cada vez más de hacer inferencias sobre la localización de la patología, los efectos de diversos tratamientos quirúrgicos o con drogas, el pronóstico en cuanto a la recuperación y las estrategias para acelerar la restauración de la función y la rehabilitación. Benton $(1959,1974)$ ha aplicado la neuropsicología clínica a los problemas del cambio comportamental, mientras que Rei$\tan (1955,1974)$ ha utilizado los procedimientos de la neuropsicología experimental y clínica y de la neurología conductual para analizar el funcionamiento cerebral de pacientes concretos. Así vemos cómo dos de los pioneros de la neuropsicología clínica acentúan funciones distintas de ésta, aunque ambos tratan de realizar una investigación comprehensiva y estandarizada de sus pacientes. Reitan ha modificado y ampliado las baterías de tests originales de Halstead, que se habían desarrollado con el objetivo de proporcionar un «índice de deterioro» de la «inteligencia biológicas, debido a su supuesta sensibilidad a la disfunción del lóbulo frontal (Halstead, 1947, 1951, Halstead y Rennick, 1966; Halstead y Wepman, 1949). En los estudios diagnósticos, la Batería de Pruebas Neuropsicológicas de Halstead para adultos (a partir de 15 años) y la de niños (de 9 a 14 años) y la Batería de Pruebas Neuropsicológicas de Reitan-Indiana para niños (de 5 a 8 años), tal como la administra Rei- 
$\tan (1969)$ han demostrado ser sensibles a una gran diversidad de localizaciones, etiologías y tipos de lesiones cerebrales (Reitan, 1956, 1959, 1962, 1964, 1966a, 1966b; Russell, Neuringer y Goldstein, 1970). La neurología, la neurocirugía y la psiquiatría han acudido tradicionalmente a la psicología clínica en busca de evaluaciones psicométricas y hasta que no haya más personas que practiquen la neuropsicología clínica, la evaluación de los pacientes con una lesión cerebral, supuesta o confirmada, seguirá realizada por los psicólogos clínicos con alguna preparación en neuropsicología. El aumento del interés por la neuropsicología clínica que se da actualmente entre los estudiantes de psicología clínica - que, por otra parte, se oponen mucho, con frecuencia, a las pruebas psicológicas formales- se debe, en gran parte, a la naturaleza internacional de los esfuerzos de investigación, que indica que ciertos descubrimientos neuropsicológicos son fundamentales para los seres humanos, prescindiendo de las influencias culturales (en Rusia Iuria, 1962; en Francia Hécaen y Ajuriaguerra, 1964; en Italia De Renzi, Faglioni y Scotti, 1970; en Noruega Klove, 1974; en Canadá y EE. UU. Milner y Teuber, 1968; en el Reino Unido Newcombe, 1969; Warrington y Weiskrantz, 1973). También resulta atractiva la variedad de sistemas conceptuales comprehensivos que se da en la neuropsicología humana (Geschwind, 1965; Luria, 1973; Pribram, 1971). Ni la validez intercultural ni los marcos teóricos están establecidos en la psicología clínica - especialmente en sus áreas más psicométricas-, exceptuando algunas áreas como la terapia de la conducta (Eysenck, 1976; Gray, 1976; Rachman, 1976; Feldman y Broadhurst, 1976) y el «biofeedback» (Dicara et al, 1975).

La diferencia principal entre el enfoque de la psicología clínica y el de la neuropsicología clínica es que en la primera se pasan pruebas a los pacientes para buscar pruebas de la existencia de un «síndrome orgánico» como, por ejemplo, la pérdida de la «actitud abstracta» (Goldstein, 1952), mientras que en la segunda se pasan pruebas buscando signos de la localización, la etiología y la naturaleza de la lesión. El psicólogo clínico supone que las diversas clases de disfunciones cerebrales provocan deficiencias similares y que las diferencias que pueda haber se deben a la gravedad de la lesión y a las características de la personalidad premórbida. De modo que, siguiendo la filosofía general de Lashley (1929), el psicólogo clínico considera que la «organicidad» es un concepto unitario y que las variaciones en la conducta se deben a que la lesión cerebral varía sólo de grado, dependiendo de la gravedad de la patología orgánica. Ciertamente es importante la amplitud y la gravedad de la lesión cerebral (Chapman y Wolff, 1959), pero los psicólogos clínicos, como Reitan (1966 a) han señalado muchos otros factores que influyen en la forma y en la naturaleza del cambio de conducta, como, por ejemplo, la condición premórbida del paciente, la causa de la lesión, la edad del paciente en el momento de ésta, la lateralidad de la dominancia motora y cerebral, la lateralidad de la lesión, su situación en el hemisferio cerebral, el intervalo entre el momento de la lesión y el de la prueba y el sexo del paciente (Lansdell, 1962, 1964, 1971; ver también Buffery, 1974, p. 228).

A los especialistas médicos que acostumbran a remitir a los psicólogos 
pacientes con lesión cerebral segura o posible, les suelen resultar más familiares los tests e inferencias de los psicólogos clínicos que los de los neurpsicólogos clínicos. Ello se debe, en parte, a que aún hay relativamente pocos neuropsicólogos clínicos y, en parte, a que la formación en psicología que se da en disciplinas como la neurología, la neurocirugía y la psiquiatría suele limitarse a unos pocos principios generales y un poco de psicometría. Además de no comprender del todo los informes de los neuropsicólogos clínicos, ni apreciar cuál pueda ser su contribución, estos especialistas médicos pueden sentir que se entra en su territorio en ciertas ocasiones, como, por ejemplo, cuando se realiza un análisis cuantitativo de las habilidades senso-motoras de un paciente que no coincide del todo con su propia impresión. También puede ocurrir que el neuropsicólogo clínico no comprenda ni respete suficientemente el poder diagnóstico del examen neurológico tradicional. Estas dificultades podrán superarse a medida que aumenten los contactos entre los especialistas médicos y los neuropsicólogos clínicos, cuyo número es cada vez mayor, pero este proceso se aceleraría si se revisasen apropiadamente los planes de estudio de medicina y psicología. Las perspectivas de obtener un beneficio mutuo son buenas si tenemos en cuenta algunas de las investigaciones que ya han emprendido especialistas médicos y neuropsicólogos clínicos en colaboración, por ejemplo, Blakemore y Falconer (1967); Gazzaniga, Bogen y Sperry (1962, 1965); Heimberger y Reitan (1961); Klonoff y Low (1974); Klonoff y Paris (1974); Penfield y Milner (1958).

Ahora vamos a examinar las contribuciones actuales y potenciales de la neuropsicología en relación con los pacientes con diversos tipos de alteraciones del sistema nervioso superior. Los lectores que se interesen especialmente por los detalles de los materiales y procedimientos de prueba pueden remitirse a los escritos de Reitan y Davison (1974) y Smith (1975).

\section{LA CONTRIBUCION ACTUAL DE LA NEUROPSICOLOGIA CLINICA}

En el examen neurológico se emplean diversas técnicas diagnósticas, por ejemplo, el electroencefalograma (EEG), el examen del fluido cerebro-espinal (FCE), los exámenes cerebrales (incluyendo el examen EMI, que es nuevo y muy sensible) y otros estudios de rayos con contraste (Bannister, 1973; Walton, 1975). La precisión diagnóstica de estas técnicas para lesiones confirmadas varía según la naturaleza de la lesión, su lugar, su amplitud y la edad. Según los informes oscila entre el 70 y el $97 \%$ (Afifi et al, 1965; Dichiro, 1962; Marttila y Heiskanen, 1970; Mc Afee y Taxdal, 1961; Rasmussen, 1970). Ciertas pruebas neuropsicológicas de lesión cerebral (revisadas por Spreen y Benton, 1965) han demostrado tener una precisión de un $80 \%$ aproximadamente, con un máximo del $94 \%$ (Yates, 1954).

La mayoría de los pacientes remitidos por el neurólogo para que se les efectúe una exploración neuropsicológica, lo han sido inicialmente por el médico general, debido a sus síntomas de disfunción motora, sensorial, lingịística y/o mental. Sin embargo, los neurocinujanos romitên a 
algunos pacientes al neurólogo (y éste al neuropsicólogo) para que evalúe más profundamente las implicaciones conductuales de la neurocirugía. Otros son pacientes psiquiátricos, cuya configuración de síntomas mentales sugiere la existencia de una ncuropatología subyacente. Cuando en los síntomas que se presentan prevalecen las alteraciones mentales éstas pueden encubrir la existencia de otros signos neurológicos de lesiones intracraneales (Chambers, 1955; Harris, 1965; Hobbs, 1963; Malamud, 1967; Olin y Weisman, 1964; Remington y Rubert, 1962; Waggoner y Bagchi, 1954; Whitty, 1956).

Los pacientes remiridos el psicólogo clínico pertenecen, hablando en un sentido amplio, a dos categorías: aquellos en que se piensa que ya se ha establecido la naturaleza y localización de la disfunción del sistema nervioso superior y aquellos en que no se ha establecido. En lo que se refiere a la primera clase de pacientes, el neuropsicólogo clínico puede proporcionar medidas objetivas de la significación funcional de la lesión neural, focal o difusa, en un momento específico de la historia de ia patología, contribuyendo así al pronóstico sobre el desarrollo de los déficits y a formular estrategias para compensarlos. En cuanto al segundo tipo de pacientes, el neuropsicólogo clínico puede ayudar al diagnóstico y contribuir a que se tome una decisión sobre si está justificado el empleo de otros procedimientos subsidiarios que pueden suponer un riesgo o resultar molestos, como, por ejemplo, la arteriografía, la ventriculografía, la mielografía, la pneumoencefalografía y las inyecciones intra-carótidas de amital-sódico.

En los casos en que se ha dado un diagnóstico de una alteración neurológica bien definida, como la corea de Huntingdon, la esclerosis múitipie, ia epiiepsia «idiopática», ia enfermedad de Paíkiñsoñn ô dê alteraciones endocrinas, como el síndrome de Cushing, el neuropsicólogo clínico puede muchas veces hacer más específico el curso de los diversos cambios mentales en relación al proceso de enfermedad. En la enfermedad de Pick o de Alzheimer y en otras demencias (Bignami, 1975), en algunos casos de tumor cerebral (Mulder, 1959) la exploración del neuropsicólogo clínico sobre los cambios de inteligencia y personalidad puede mostrarse más sensible a la manifestación precoz de la afección cerebral que el examen neurológico (ver la revisión de Reitan, 1976). Cuando se ha establecido la naturaleza y localización de una lesión cerebral traumática, el neuropsicólogo clínico puede proporcionar medidas objetivas de la significación funcional de la lesión y líneas base para evaluar los efectos a largo plazo (Walker, Caveness y Critchley, 1969), el curso de la rehabilitación (Hook, 1969) o la influencia de diversos regímenes terapéuticos (Lishman, 1966, 1968, 1973; Reynell, 1943, 1944). El grado de deterioro funcional y su duración son criterios importantes y prácticos de la gravedad del trauma cerebral, ya que el seguimiento neuropsicológico de niños con lesiones de cabeza (revisado por White, 1975), por ejemplo, ha demostrado que se retarda el desarrollo mental y aparecen crisis, hiperkinesia y alteraciones de conducta (Black et al., 1969; Richardson, 1963).

Para poner un ejemplo de la contribución actual de la neuropsicología clínica revisaremos algunas de las técnicas que se utilizan en las 
exploraciones sobre las funciones de las estructuras subcorticales (ejemplo, Ojemann, 1975 a) y corticales (ejemplo, Milner, 1972) y algunos de los resultados de dichas exploraciones.

La publicación sobre «Cerebro y lenguaje», editada por Ojemann (1975 a) contiene varias investigaciones sobre la relación entre las lesiones talámicas o la estimulación talámica y las destrezas lingüísticas en pacientes que se someten a una operación quirúrgica para aliviar sus alteraciones motrices. Riklan y Cooper (1975) revisan los estudios psicométricos del lenguaje de pacientes con la enfermedad del Parkinson que fueron sometidos a una talamotomía ventrolateral para tratar su temblor o rigidez, o a una operación pulvinar para aliviar la espasticidad o el dolor secundarios a un golpe (ver también Cooper, Amin. Chandra y Waltz, 1973). Estos estudios indican que los núcleos talámicos, y en concreto los núcleos ventrolaterales y pulvinares, intervienen en habilidades lingüisticas que exigen fluidez verbal. El tálamo izquierdo parece estar más implicado en la fluidez verbal que el derecho, pero esta lateralización no se considera una dicotomía absoluta (como ocurre con la dominancia verbal para el lenguaje). Se piensa que el tálamo interactúa con los sistemas cerebrales, troncoencefálicos, de los ganglios basales, y posiblemente con el sistema límbico, al servicio de las habilidades lingüisticas y también con otros mecanismos sensomotores y de «arousal», específicos que están al servicio de la conducta verbal. De los 123 pacientes con la enfermedad de Parkinson, y con otras alteraciones motrices, estudiados por Darley, Brown y Swenson (1975) la cuarta parte mostraron variaciones en determinadas habilidades lingüísticas después de que se les efectuara talamotomia, palidectomía o una palidotomia-talamotonía mixta. Los cambios eran mayores después de la talamotomía izquierda, talamotomías múltiples y procedimientos mixtos. Mohrs, Watters y Duncan (1975) describen cuatro casos de autopsia y a dos pacientes vivos con hemorragias talámicas izquierdas que tenían historias de recaídas en estados de parafasia que podían diferenciarse claramente de otros síndromes afásicos. Sin embargo, Van Buren (1975) previene contra el peligro de dar una importancia excesiva al papel del tálamo izquierdo en el habla, a la luz de su revisión sobre los efectos de las lesiones intratalámicas y de la estimulación talámica. De forma similar, Schaltenbrand (1975) señala que, además de las interrupciones y compulsiones del habla que se producen como resultado de la estimulación del tálamo ventro-oral y posterior, el habla puede detenerse totalmente por implicación del cuerpo calloso anterior o interrumpirse debido a las confusiones de pensamiento que se dan cuando se estimula el cuerpo calloso posterior. Ojemann (1975 b) ha revisado otras investigaciones sobre los efectos de la estimulación del tálamo (Ojemann y Fedio, 1968; Ojemann, Fedio y Van Buren, 1968). Mientras se estimulaba el tálamo ventrolateral y en el momento de una talamotonía estereotáctica, así como dos días después de la operación, se pasaron pruebas de denominación de objetos, àritmética mental y memoria a corto plazo. Cuando se estimulaba el lado izquierdo del tálamo se encontraba una deficiencia en la denominación de objetos que no se hallaba al estimular la parte derecha. La repetición verbal se evocaba al estimular el núcleo anteriolateral ventrolateral, y la persevera- 
ción y dificultades de denominación cuando se estimulaban porciones más centrales del núcleo ventrolateral. Ojemann sugiere que la estimulación talámica izquierda evoca una «respuesta específica de alertas que dirige la atención, a la vez que bloquea la recuperación del material de la memoria. Esta respuesta de alerta afecta tanto a la memoria a corto plazo como a la memoria a largo plazo y está correlacionada con el grado de dificultad para denominar objetos que los pacientes tienen después de una operación. Fedio y Van Buren (1975) encontraron pruebas de que existe una asimetría lateral en la organización de las funciones verbales y espaciales en el tálamo. Mediante la estimulación del núcleo pulvinar izquierdo se provocó una disfasia transitoria y una amnesia retrógada del material verbal reciente. Sin embargo, cuando se estimulaba el núcleo pulvinar derecho se disminuía la discriminación y el reconocimiento de configuraciones estimulares visuales complejas. De forma similar, Vilkki y Laitinen (1974) encontraron que con la talamotomía izquierda se deterioraba ia eficacia verbai a nivel receptivo y expresivo, mientras que con la talamotomía derecha se disminuiá la precisión con que se realizaba una tarea de asociación de caras. Ojemann (1974) ha demostrado que los efectos de la estimulación talámica derecha se diferencian sutilmente de los de la estimulación izquierda en una tarea de aritmética mental. Ojemann (1974) relaciona sus resultados con la hipótesis de la existencia de una «tespuesta especcífica de alerta» controlada por el tálamo izquierdo. Cuando se estimulaba el tálamo izquierdo aumentaba el ritmo con el que el sujeto contaba «hacia atrás» de tres en tres y aumentaba el número de errores, pero no variaba la latencia de identificación del número inicial. Cuando se estimulaba el tálamo dêrechó dismininía la velocidad con que contaba el sujeto, incrementándose los errores y la latencia.

Estos estudios indican que es asimétrica la organización funcional de las habilidades lingüísticas y no lingüísticas incluso al nivel del tálamo lateral. Lo que es necesario descubrir es hasta qué punto se correlacionan, en cada individuo, la asimetría funcional talámica con la asimetría funcional cerebral. También sería interesante saber si existen o no diferencias entre uno y otro sexo en cuanto a las estructuras talámicas - diferencias similares quizá a las que han descubierto en las estructuras cerebrales Witelson y Pallie (1973) - y que pudieran relacionarse con las pruebas que demuestran que las mujeres tienen mayor fluidez verbal (revisados por Buffery y Gray, 1972; Hutt, 1972). Además, estas variables psicológicas, así como la posibilidad de una "dominancia talámica» deberían examinarse, midiendo, al mismo tiempo, la naturaleza de la disfunción motora y el grado de ésta antes del tratamiento, durante el tratamiento y después de él (Bowen, Hoehn y Yahr, 1972; Flowers, 1975; Marsden, 1975; Marsden y Parker, 1973; Parker y Mardsen, 1973; Perret, 1970; Levita y Rikland, 1973).

La asimetría del funcionamiento cerebral también constituye un factor clave para dar una interpretación neuropsicológica de los efectos de las lesiones corticales localizadas en el hombre. En los pacientes que se someten a operaciones cerebrales para aliviar la epilepsia, la resección de tejidos del lóbulo temporal anterior del hemisferio cerebral dominante 
en la función lingüística (que es, normalmente, el izquierdo) se acompaña de una disminución del aprendizaje y la retención de los materiales fáciles de verbalizar (FV) (Meyer y Yates, 1955; Milner, 1958), prescindiendo de que la presentación sea leída o auditiva (Blaklmore y Falkoner, 1967; Milner, 1967) y de que se les pida que recuerden o que reconozcan (Milner y Teuber, 1968).

Sin embargo, esta lesión del hemisferio cerebral dominante en el lenguaje no implica un deterioro de la percepción o la memoria de lugares, caras, melodías o dibujos sin sentido. A la inversa, la lobotomía temporal del hemisferio cerebral no dominante en la función lingüística (normalmente el derecho) deja intactas la percepción y memoria verbales, pero implica un deterioro del reconocimiento y el recuerdo de configuraciones visuales y auditivas difíciles de verbalizar (D. V) (Kimura, $1963 a$; Milner, $1868 a, 1968 b$; Shantwerler, 1966; Warrington y James, 1967) y también supone un deterioro del aprendizaje de laberintos con punzón, tanto si se guía por la información visual como si se rige por la propioceptiva (Corkin, 1965; Milner, 1965). También se dan efectos de lateralidad cuando se realiza una lobotomía frontal en el hemisferio cerebral dominante en el lenguaje (normalmente el izquierdo). Luria (1958, 1962) consideraba que este déficit supone una pérdida del monólogo espontáneo y se observa tanto en la escritura como en el habla (Benton, 1968; Milner, 1964; Ramier y Hècaen, 1970). La lobotomía frontal izquierda o derecha puede dar lugar a un deterioro de la ejecución en la tarea de ordenación de cartas de Winsconsin (Milner, 1963, 1964), en la que el paciente tiene que variar sus estrategias de ordenación siguiendo señales verbales, y también en los laberintos con punzón, tanto visuales (Milner, 1965) como táctiles (Corkin, 1965), en los que el paciente debe modificar las direcciones siguiendo señales no verbales de carácter auditivo. En cualquier caso, con la lobotomía fronral en el hemisferio cerebral dominante (normalmente el izquierdo: el foco del déficit suele ser el lóbulo frontal dorsolateral izquierdo) se produce un deterioro más duradero en la ordenación de cartas. Y a la inversa, la lobotomía frontal en el hemisferio cerebral no dominante en el lenguaje (normalmente el derecho) produce un deterioro más duradero en el aprendizaje de laberintos, sobre todo cuando el sujeto se sirve de guías propioceptivas para realizarlo. Hay datos sin publicar sobre pacientes con lesiones en el lóbulo frontal (Prisko, citado por Milner, 1964) y sobre otros pacientes con lesiones en el lóbulo frontal y en el temporal (Corsi, citado por Milner, 1971) que indican que con la lobotomía frontal se deteriora la capacidad de discriminar la recencia relativa de los sucesos (ver también Pribram y Tus, 1967; Yntema y Trask, 1963), mientras que con la lobotomía temporal se deteriora la capacidad de consolidar la experiencia con el paso del tiempo; el grado de deterioro está directamente relacionado con el grado de excisión del hipocampo (Milner, 1970). Estos deterioros son específicos de determinados materiales (por ejemplo, de los materiales FV o DV), dependiendo de la lateralidad de la lesión de relación con la asimetría funcional de los hemisferios cerebrales. El deterioro de la consolidación de ciertos materiales que se da después de la lobotomía 
temporal unilateral debe distinguirse de la amnesia global asociada con los daños bilaterales del hipocampo (Milner, 1959; Milner, Corkin y Teuber, 1968; Scoville y Milner, 1957; Warrington, 1971). En pacientes epilépticos se descubrió que había un déficit de la búsqueda visual después de una lobotomía frontal derecha o izquierda y que se daba un deterioro de la comparación con demora de estímulos visuales fáciles de verbalizar (FV) después de la lobotomía temporal izquierda y de los difíciles de verbalizar después de la derecha (Buffery, 1969, 1974). En este caso se da una «doble disociación» (Teuber, 1955) de un déficit de atención después de la lobotomía frontal unilateral, que se diferencia del déficit de memoria después de una lobotomía temporal unilateral. Esta «doble disociación» en el hombre es similar a la que se encuentra en el cinocéfalo después de la lobotomía bilateral (Buffery, 1964, 1965, 1966, 1967).

Meier (1974) describe otros intentos de comprender, desde la neuropsicología clínica, los efectos inmediatos de diversas lesiones cerebrales, asi como sus implicaciones a largo plazo. Los problemas específicos de las afasias, apraxias, agnosis y amnesias están bien revisadas en los volúmenes 3 y 4 del Manual de Neuropsicología Clínica, editado por Vinken, P. S. y Bruyn, G. W. (1969 a, 1969 b). Antes de pasar a establecer cuál puede ser la contribución potencial de la neuropsicología clínica (contribución en la que también tiene gran relevancia el concepto de dominancia cerebral) hay que reclacar que el trabajo con pacientes comisurotomizados (revisado por Sperry, Gazzaniga y Bogan, 1969) y sobre los lóbulos parietales (Critchley, 1953; Hécaen y Assal, 1970; Semmes, Weinstein, Ghent y Teuber, 1955; Warrington, 1969) ha dejado establecido que la asimetría hemisfërica de una determinada función es un fenómeno relativo y no absoluto; es decir, el hemisferio cerebral no dominante en el habla (normalmente el derecho) contribuye a la comprensión verbal y el lóbulo parietal dominante (normalmente el izquierdo) contribuye a la habilidad espacial (ver también, Buffery, 1968).

\section{LA CONTRIBUCION POTENCIAL DE LA NEUROPSICOLOGIA CLINICA}

A partir del trabajo de Wolpe (1958) los psicólogos clínicos han añadido varias técnicas terapéuticas (revisadas en Feldman y Broadhurst, 1976) a sus métodos psicométricos (revisados por Cronbach, 1970). El neuropsicólogo clínico está a punto de lograr un avance similar, no en la terapia de conducta, sino en la reinstauración de las funciones cerebrales. Los resultados de las investigaciones sobre el desarrollo y la regeneración de los sistemas nerviosos de diversas especies (revisados por Gaze y Keating, 1974) tienen una relevancia considerable para los intentos de restaurar funciones cerebrales o de acelerar el ritmo de compensación de lesiones cerebrales en el hombre (por ejemplo, Luria, 1948; Luria et al, 1969). Estos aspectos de la investigación pura y aplicada sobre la especificidad y plasticidad del sistema nervioso convergen en la neuropsicología, estimulando las innovaciones técnicas (por ejemplo, Buffery, 1971a) y la formulación de teorías de valor heurístico (por ejemplo, Kinsbourne, 1974 a, 1974 b) 
que, tomadas en conjunto, pueden contribuir a desarrollar los instrumentos terapéuticos con que cuenta el clínico (Stein, Rosen y Butters, 1974).

Cuando una persona sufre un daño cerebral unilateral debido a traumatismo, aneurisma, hemorragia, neoplasma, epilepsia, etc., el grado y ritmo de compensación de la función depende principalmente de la gravedad y localización de la lesión y de la edad y el sexo del paciente. Esta compensación se da mediante la reorganización de la topografía funcional del hemisferio cerebral dañado o mediante la transferencia de la función deteriorada al hemisferio cerebral intacto o por una combinación de estos dos procesos. Cuando se enfrenta a un paciente concreto con lesión cerebral, el neuropsicólogo clínico debe ser capaz de descubrir la naturaleza idiosincrática de la compensación que se está llevando a cabo y luego promover esa compensación utilizando técnicas desarrolladas en los laboratorios de psicología experimental que faciliten la estimulación del cerebro -inicialmente unilateral- con materiales FV o DN presentados en una modalidad visual, auditiva y/o tactil (técnicas revisadas y utilizadas por Buffery y Gray, 1972; Buffery, 1974, 1976). La información que proporciona el análisis y el pronóstico del neuropsicólogo clínico acerca de la patología funcional del cerebro dañado, junto con la influencia de la terapia de la función cerebral, puede suponer una contribución valiosa para diseñar un programa de reahabilitación específica para cada paciente - un programa basado en la medición neuropsicológica concomitante con la restauración en el paciente de la función cortical superior y en estrecha cooperación con neurólogos, fisioterapeutas, terapeutas ocupacionales, nodrizas y asistentes sociales-, diseñado como un programa integrado y equilibrado de actividad para promover el bienestar médico, mental y social del paciente.

Por ejemplo, si un paciente sufre diversos deterioros en sus habilidades lingüísticas debido a una lesión cerebral unilateral en el hemisferio izquierdo dominante, entonces (además de realizar la determinación más usual de su inteligencia y personalidad) el neuropsicólogo clínico podría tratar de realizar el análisis y la terapia que se exponen a continuación: como cada hemisferio cerebral sirve, primariamente, a su campo visual contralateral, y a su oído y miembros contralaterales, es posible comparar la eficacia del procesamiento de estímulos fáciles de verbalizar (FV) por parte del hemisferio cerebral izquierdo, que está dañado, con la del derecho intacto mediante la presentación de materiales apropiados a las modalidades visual, auditiva y/o tactil con técnicas de exposición taquitoscópica a un hemicampo visual (Kimura, 1966; Mishkin y Forgays, 1952), escucha dicótica (Broadbent, 1954; Kimura, 1963 b, 1967; Milner, 1962) y diversas tareas de comparación de manos (Buffery, 1971 b; Buffery y Gray, 1972; Milner, 1971; Milner y Taylor, 1972; Witelson, 1974). Después de determinar la eficacia relativa de los dos hemisferios cerebrales en variaciones intramodales y transmodales de dichas tareas (Hines y Satz, 1974) podría calcularse una primera medida de dominancia verbal con el material FV (ver «dominancia cerebral verbal» DCV en Buffery, 1976). Después de una semana o así, podría calcularse una segunda medida de DCV a partir de los resultados de un retest con tareas similares FV. Ia 


\section{Estudios}

comparación de la primera medida de DCV con la segunda indicaría si la compensación de las habilidades lingüísticas está siendo servida primordialmente por el desarrollo funcional del hemisferio cerebral izquierdo dañado o por el hemisferio derecho intacto (Kinsbourne, 1974 b; Nielsen, 1946). Los resultados de este análisis ayudarían a determinar si la terapia debe concentrarse en el hemisferio cerebral ipsilateral o contralateral al daño. En la mayor parte de las circunstancias, la terapia deberá concentrarse en el hemisferio cerebral más comprometido con la actividad compensatoria, es decir, será una «terapia de la función cerebral» para acelerar la pauta de compensación observada. Sin embargo, pueden darse circunstancias en que la terapia de la función cerebral deba utilizarse para iniciar la compensación o para inhibir, o incluso invertir, la pauta observada de compensación. En Isaacson (1975) y Le Vere (1975) pueden encontrarse enfoques alternativos.

Las sesiones regulares diarias de terapia de la función cerebral deberían incluir estimulación intermodal y transmodal deí hemisferio cerebrai elegido con material FV, presentado en una batería de tareas que sirvan, en parte, para practicar las habilidades lingüísticas deterioradas y en parte para reciclar las intactas. Por ejemplo, el reconocimiento visual de palabras o de dibujos FV, la comparación con demora de sonidos de habla emparejados, la discriminación al tacto de formas de letras. La naturaleza de la respuesta del paciente (hablada, escrita, respuesta no verbal de apretar un botón, etc.) debe decidirse también a la luz de la necesidad de equilibrar el ejercicio de las habilidades deterioradas e intactas. Si se elige el hemisferio cerebral derecho para realizar la terapia de función cerebral el siguiente ejemplo puede servir para ilustrar una tarea intermodal, avanzada, quic cxijc quic sc den respuestas habladas. En cada ensayo se presentan simultáneamente diferentes palabras a través de las vías de las modalidades visual, auditiva y tactil para realizar un procesamiento inicial por el hemisferio cerebral derecho, es decir: la exposición taquitoscópica de una palabra impresa (por ejemplo, «RAPIDO») al hemicampo visual izquierdo, la presentación dicótica de una palabra hablada (por ejemplo, «LENTO») al oído izquierdo en conflicto con un «ruido blancon al derecho y la presentación de una figura de una palabra (por ejemplo, "VELOZ») para que sea palpada por la mano izquierda en conflicto con la respuesta de palpar una forma irrelevante con la mano derecha (para descripciones y fundamentación de estas técnicas, ver Buffery, $1971 a, 1971$ b, 1974, 1976; Buffery y Gray, 1972). Al paciente se le pide que nombre aquella palabra - de las tres presentadas- que no tiene un significado similar a las otras dos, es decir, «LENTO». En cada ensayo se dicen palabras nuevas y se varía aleatoriamente la modalidad de la "palabra que sobra» a lo largo de la tarea, con la limitación de que cada una de las modalidades se utiliza para presentar «la palabra que sobras en un tercio de los ensayos.

Esta tarea exigiría un trabajo considerable al hemisferio cerebral derecho intacto del paciente que tendría que realizar análisis intramodales y transmodales del material FV. Hay investigaciones recientes sobre la relación entre la naturaleza y la pauta de estimulación sensorial, por una parte, y el crecimiento neuronal y el desarrollo funcional por otra (por ejemplo, 
Berry, 1973; Hirsch y Jacobson, 1975; Sperry, 1971; Terzadze, 1974; Trevarthen, 1973). Estas investigaciones apoyan la creencia de que estas estrategias terapéuticas neuropsicológicas (ver también Gazzaniga, 1970) pueden acelerar la actividad compensatoria y contribuir así a la restauración de la función cortical superior después de un daño cerebral. Para acelerar la compènsación de habilidades no verbales deterioradas (por ejemplo, de carácter espacial) producidas después de un daño del hemisferio cerebral no dominante en el habla (ver hemisferio cerebral «menor», Joynt y Goldstein, 1975) pueden utilizarse tareas similares, pero utilizando materiales difíciles de verbalizar (DV). Hay diversos tipos de afasias y disfasias (Wyke, 1971) que también pueden beneficiarse de una estrategia neuropsicológica de terapia más estructurada (Darley, 1975).

Para que la neuropsicología clínica incremente su contribución diagnóstica y terapéutica a la medicina, debe alejarse de los «mapas cerebrales» simplistas, estáticos y uniformes (revisados por Young, 1970) que se utilizan como «plantillas» para encajar en ellas las puntuaciones psicométricas, y acercarse a la comprensión del desarrollo sutil, dinámico e idiosincrático de la topografía funcional del cerebro individual normal y anormal (Buffery, 1974)... lo que, de hecho, está más cerca de la ciencia neuropsicológica promovida por Pribram (1954) y Luria (1967). La presencia de una diasquisis (Von Monakow, 1911, 1914) o, como lo denominó Reese (1959), del «efecto a distacía» de una lesión, por el cual pueden perturbarse las funciones de estructuras intactas incluso contralaterales al foco de lesión por influencia de una patología, puede dar lugar a que se interprete de forma inadecuada la ejecución del paciente, y a que se confunda el diagnóstico y la localización de la lesión cerebral. De forma similar, el clínico debe guardarse de concebir la dominancia como si fuera una asimetría funcional absoluta de los hemisferios cerebrales. Muchas veces puede establecerse la función lingüística en el hemisferio cerebral «menor» (Jackson, 1874; Zangwill, 1967), cuando está dañado el hemisferio cerebral "mayor» responsable del habla. Es posible que existan diferencias entre uno y otro sexo en cuanto al grado de asimetría de la función cerebral (Buffery, 1970; Buffery y Gray, 1974; Witelson y Pallie, 1973). Además Zangwill (1960) ha observado que los pacientes zurdos de mano y ambidextros se recuperan mejor que los diestros de la afasia debida a un trauma cerebral izquierdo y Oldfield (1971) ha demostrado que se da una incidencia mayor en zurdos entre los hombres que en las mujeres. Todo ello tiene implicaciones en cuanto al pronóstico de la recuperación y las estrategias terapéuticas consiguientes a un daño cerebral unilateral. En todo caso, las investigaciones más recientes sobre la relación entre la dominancia cerebral y el sexo (Fairweather, 1976; Lukianowicz, 1974; Maccoby y Jacklyn, 1975) y la lateralidad (Brown y Hécaen, 1976; Buffery, 1976; Satz, Achenback y Fennel, 1967) alientan al neuropsicólogo clínico a probar directamente el grado de asimetría de la función cerebral, en vez de estimarla basándose en el sexo o preferencia manual del paciente. 


\section{OBSERVACIONES FINALES}

La investigación clínica de los efectos de diversas lesiones cerebrales sobre las funciones psicológicas del hombre (por ejemplo, Conkey, 1938) se hace más penetrante cuando se mira desde las perspectivas ontogenética y filogenética. La neuropsicología evolutiva (por ejemplo, Boll, 1974; Dikman, Matthew y Hazley, 1975; Fitzhugh y Fitzhugh, 1965; Taylor, 1969) nos proporciona la perspectiva ontogenética. La neuropsicología comparada (por cjemplo, Dean, 1976; Piercy, 1964) la filogenética. Así, por ejemplo, la investigación clínica de pacientes adultos con amnesia (por ejemplo, Shallice y Warrington, 1970; Warrington y Weiskrantz, 1973), incluyendo los que padecen la enfermedad de Korsakoff (por ejemplo, Butters y Cermak, 1974; Seltzer y Benson, 1974) se ha hecho más sofisticada en el aspecto teórico y metodológico por referencia no sólo a otros estudios de adultos amnésicos (revisados por Flowers, 1972), sino también a los estudios de la memoria de niños con daños cerebrales (revisados por White, 1975) y de animales con lesiones cerebrales (revisados por Iversen, 1973). Para que una teoría de la función cerebral humana tenga relevancia clínica tiene que tratar de integrar la experiencia de los neuropsicólogos experimentales, evolutivos y comparados. Kesner (1975) trata de hacerlo en lo que se refiere a los problemas de almacenamiento y recuperación de la memoria, pero, por ejemplo, los datos sobre el papel de los lóbulos parietales en la mediación de las asociaciones intermodales (por ejemplo, Butters y Brody, 1968) sólo se apoyan en las teorías del procesamiento de la información (por ejemplo, Freides, 1974) y éstas suelen preocuparse poco de la influencia de determinados variables neuropsicológicos como, por ejemplo, de la influencia de "la asimetría funcional en las habilidades intermodales (Buffery, 1971 a, 1971 b, 1976; Buffery y Gray, 1972; Hines y Satz, 1974).

Cuando el neuropsicólogo evalúa o trata a un paciente con daño cerebral es vital que tome en consideración la asimetría funcional, tanto en lo que se refiere a la función como a la estructura del cerebro. Sin un conocimiento claro del grado de asimetría del funcionamiento cerebral del paciente resulta imposible interpretar las puntuaciones de los tests en relación a la localización de la lesión cerebral y formular una estrategia para la terapia de la función cerebral. Hay que diferenciar el procesamiento de uno y otro hemisferio cerebral con materiales FV y DV mediante técnicas tales como la exposición taquitoscópica a los hemicampos visuales y/o la escucha dicótica. Las medidas de preferencia manual y dominancia ocular no predicen suficientemente por sí mismas la dominancia cerebral - se necesitan métodos más directos- (Buffery, 1976). Los métodos médicos actuales para determinar la dominancia cerebral en el habla son la inyección intracarótida de amital sódico (Wada y Ramussen, 1960) y, en ocasiones, la ECT unilateral (Warrington y Pratt, 1973). En un próximo futuro estos métodos traumáticos para probar la dominancia cerebral en el habla pueden reemplazarse por otras técnicas neuropsicológicas más sensibles (por ejemplo, Buffery, 1971; Kinsbourne, 1972). Los libros de Gazzaniga (1970), Dimond (1972), Dimond y 
Beaumont (1974) y Kinsbourne y Smith (1974) recogen bien los datos, las teorías y la metodología sobre la asimetría de la función y estructura del cerebro.

El interés actual por las bases biológicas del lenguaje y su influencia en el desarrollo cognitivo en primates parte de dos publicaciones de 1949: «Organization of Behavion, de Donald Hebb, y «Concept of Mind», de Gilbert Ryle. Poco después se examinó de una forma nueva y profunda la estructura del lenguaje (Chomsky, 1957, 1959) y se reconsideró el concepto de dominancia cerebral (Zangwill, 1962). Se desarrollaron nuevas técnicas (por ejemplo, Garner, 1962) para facilitar el estudio de destrezas cognitivas de nivel superior como la formación de conceptos (Bruner, Goodnow y Austin, 1956) y la percepción del lenguaje (Broadbent, 1958). Al perder vigencia el conductismo simplista surgieron teorías que trataban de explicar la orquestación de las pautas de comportamiento (Miller, Galanter y Pribram, 1960) y el mejorar las técricas electrofisiológicas comenzó a apreciarse la contribución del sistema nervioso al impulso (Olds y Milner, 1954) la atención (Sokolov, 1960) el condicionamiento y la habituación (Hernández. Peón y Brust-Carmona, 1961), la percepción (Hubel y Weisel, 1959) y el aprendizaje (Deutsch, 1960). Las expectaciones del organismo que aprende llegaron a convertirse en un concepto respetable (Mowrer, 1960), y en junio de 1963 se publicó el primer número de la revista internacional «Neuropsychologia». En «Fundamentos biológicos del lenguaje» del último E. H. Lenneberg (1967) la filosofía y la filosofía contemporáneas del lenguaje convergen en el contexto de la psicología cognitiva (Neisser, 1967). El trabajo ecléctico y seminal de Lenneberg ha animado a muchos neuropsicólogos a enfrentarse a un aspecto del problema mentecerebro investigando la relación entre la asimetría funcional y el desarrollo cognitivo en pacientes normales o con daños cerebrales así como en animales. Yeni-Komshian y Bension (1976) han encontrado una asimetría funcional en la estructura de los lóbulos temporales del cerebro del chimpancé, pero no en el del mono rhesus. Quizá la existencia de un substrato neuroanatómico de la asimetría de la función cerebral constituye un prerequisito de la adquisición del lenguaje (ver Buffery, $1971 b$; Buffery y Gray, 1972; Zangwill, 1962), ya que la asimetría de la estructura cerebral que se encuentra en los chimpancés es similar a la encontrada en adultos humanos (Geschwind y Levitsky, 1968) y neonatos (Witelson y Pallie, 1973) y los chimpancés, a diferencia de los monos thesus, pueden adquirir ciertas habilidades lingüísticas (Gardner y Gardner, 1969; Premack, 1971; revisado por Linden, 1976). El trabajo de Paivio (1971) sobre las imágenes nivela el sesgo hacia las habilidades verbales y proporciona una fuente útil de ideas y experiencias teóricas y prácticas para diseñar estrategias para compensar las deficiencias de memoria.

En esta revisión y previsión de la neuropsicología clínica han jugado un papel preeminente los conceptos de asimetría de la función cerebral y dominancia cerebral en el cerebro humano normal (Berlutzi, 1974) y en el de pacientes con daños cerebrales (Buffery, 1974). A través de la comprensión en profundidad de estos conceptos, el clínico no sólo podrá mejorar sus determinaciones, sino que habrá dado el paso para propor- 
cionar una terapia de la función cerebral. Muchas psicopatologías tienen correlatos neurofisiológicos (Hernández Peón, 1975), pero las estrategias contempladas de terapia neuropsicológica para el paciente con daño cerebral se refieren más a la neurofisiología sana que a la patológica -a lo que el paciente «puede hacer» más que a lo que «no puede hacer - se centran en establecer habilidades más que en medir su grado de destrucción. Las pruebas de la plasticidad del cerebro humano y de su capacidad de regeneración (revisadas por Gaze y Keating, 1974) y la evidencia de que en el cerebro humano se da menor diferenciación funcional y mayor equipotencialidad que en el animal (Bartlett y John, 1973; John y Kleinnann, 1975) demuestran que el paciente cuenta con la posibilidad real de que se acelere, o incluso de inicie un desarrollo funcional compensatorio, después de un daño cerebral, posibilidad para la que ya contamos con metodología y aparatos suficientes.

Cuando el neuropsicólogo experimental prueba la hipótesis de que se da una interacción hemisférica en la atención (Kinsbourne, 1973) o el neurólogo conductual investiga la dominancia cerebral de la conciencia (Rosadini y Rossi, 1967) o el neuropsicólogo clínico trata de profundizar en las intuiciones de Penfield (1965) «condicionando al lenguaje el cortex no comprometido» pueden enriquecer su estudio y su resultado a través de la medida de las latencias de las respuestas correctas e incorrectas. La medida de las latencias de respuesta ha sido durante demasiado tiempo la variable acenicienta de la psicología clínica (sobre la revisión y el uso de los tiempos de reacción y latencias de respuesta ver Fairweather, 1974, y Umiltá, Snyder y Snyder, 1972).

Illich (1976) ha puesto en tela de juicio las implicaciones de la medicina. Clare (1976) ha reconsiderado las direcciones de la psiquiatria, y muchos otros han sido menos perspicaces en sus críticas a la práctica médica y psiquiátrica establecida. En este clima de crítica y de disensión la psicología puede constituir una fuerza de integración de las diversas disciplinas médicas (Rachman y Philips, 1975) y la neuropsicología clínica puede estar en una situación especialmente buena para constituir un puente entre la neurología y los. aspectos sociales del cuidado de los pacientes. A través de su teoría, su metodología y su instrumental, la neuropsicología clínica puede constituir el foco del tratamiento interdisciplinario de los pacientes con alteraciones del sistema nervioso central: su preocupación mayor es la restauración de la función del cerebro, sin limitarse sólo a la determinación de su disfunción.

\section{RECONOCIMIENTOS}

El autor desea expresar su gratitud a la señorita Anna Wright y al profesor Robert Milburn por su ayuda en la preparación de este trabajo, y a la señorita Christine Robson por promover su conclusión. 


\section{Referencias}

AFIFI, A. K.; MORRISON, R. R.; SAKS, A. L., y EVANS, T. C. (1965) A comparison of chlormerodrin HG-203 scintiencephaloscanning with neuro-radiology and electroencephalography for the localization ofintracranial lesions, Neurology (Minneap.), 15, 56-63.

BANNISTER, R. (1973), Brain's Clinical Neurology (fourth ed.), Oxford University Press, Londres.

BARTLETT; F., y JOHN, E. R. (1973), Equipotentiality quantified: The anatomical distribution of the engram, Science, 181, 764-767.

BEKHTEREV, V. M. (1905-1907), Fundamentals of Brain Function, 7 volúmenes (St. Petersburg).

BENTON, A. L. (1959), Right-left Discrimination and Finger Localization: Development and Patbology, lloeber, Nueva York:

BENTON, A. L. (1968), Differential behaviotal effects in frontal lobe diseases, Neuropsychologia, 6, 53-60.

BENTON, A.L. (1974), Clinical neuropsychology of childhood: An overview, en Reitan, R. M. \& Davison, L. A. (eds.), Clinical Neuropsychology: Current Status and applications, V. II. Winston \& Sons, Washington, D. C.

BERLUCCHI, G. (1974), Cerebral dominance and interhemispheric communication in normal man, Schmitt, F. O. (ed.), The Neurosciences: Ard Study Program, M.I.T. Press, Cambridge, U.S.A.

BERRY, M.; HOLLINGWORTH, T.; FLINN, R., y ANDERSON, E. M. (1973), Morphological correlates of functional activity in the nervous system, en Ansell, G. B. \& Bradley, P. B. (eds.), Macromolecules and Bebaviour, The Macmillan Press Ltd., Londres y Basingstoke.

BIGNAMI, A. (1975), Out present knowledge of the pathology of dementias, in Williams, D (ed.), Moderns Trends in Neurology, 6, Butterworths, Londres.

BINET, A., y Simon. T. (1905), Méthodes nouvelles pour le diagnostic du niveau intellectual des anormaux, L'anée Psychologique, 11, 191-244.

BIRCH, H. G. (1964), The biological and social aspects, en Birch, H. G. (ed.), Brain Damage in Children, Williams \& Wilkins, Baltimore.

BLACK, P.; JEFFRIES, J. J.; BLUMER, D.; WELLNER, A., y WALKER, A. E. (1969), The post traumatic syndrome in children, en Walker, A. E.; Caveness, W. F., \& Critchley, M. (eds.), The Late Effects of Head Injury, Charles C. Thomas, Springfield, Illinois.

BLAKEMORE, C. B., y FALCONER, M. A. (1967), Long-term effects of anterior temporal lobectomy on certain cognitive functions, J. Neurol, Neurosurg, Psychiat., 30, 364-367.

BOLL, T. J. (1974), Behavioral correlates of cerebral damage in children aged 9 through 14 , en Reitan, T. M. \& Davison, L. A. (eds.), Clinical Neuropsychology: Current Status and Applications, V. H. Winston \& Sons, Washington, D. C.

BOWEN, F. P.; HOEHN, N. M., y YAHR, M. D. (1972), Cerebral dominance in relation to tracking and tapping, performance in patients with parkinsonism, Neurology (Minneap), 22, $32-39$

BROADBENT, D. E. (1954), The role of auditory localization in attention and memory span, J. Exp. Psychol., 47, 191-196.

BROADBENT, D. E. (1958), Perception and Communication, Pergamon, Nueva York.

BROWN, J. W., y HECAEN, H. (1976), Lateralization and language representation, Neurology (Minneap), 26, 183-189.

BRUNER, J. S.; GOODNOW, J. J., y AUSTIN, G. A. (1956), A Study of Thinking, John Wiley \& Sons, Inc.. Nueva York, Londres.

BUFFERY, A. W. H. (1964), The effects of frontal and temporal lobe lesions upon the behaviour of baboons, Tesis doctoral sin publicar, Universidad de Cambridge.

BUFFERY, A. W. H. (1965), Attention and retention following frontal and temporal lesions in the baboon, Proc. 73rd Ann. Conv. Amer. Psychol. Assoc. (Chicago), 103-104. American Psychological Association, Washington, D. C.

BUFFERY, A. W. H. (1966), Some effects of bilateral frontal and temporal lesions upon the behaviour of baboons, Actas 18 Cong. Intern. Psicol. (Moscú), Symposium 10, Frontal Lobes and Regulation of Behaviour, 77-83.

BUFFERY, A. W. H. (1967), Learning and memory in baboons with bilateral lesions of frontal or inferotemporal cortex, Nature, 214, 1054-1056.

BUFFERY, A. W. H. (1968), Evidence for the asymmetrical lateralization of cerebral function, Bull. Brit. Psychol. Soc., 21, 29.

BUFFERY, A. W. H. (1969), Some effects of unilateral lesions in the temporal and frontal lobes 
upon the intelligence. memory and attention of epileptics. Actas 19 Cong. Intern. Psicol. (Londres), 234.

BUFFERY, A. W. H. (1970), Sex differences in the development of hand preference, cerebral dominance for speech and cognitive skill, Bull. Brit. Psychol. Soc.. 23. 233.

BUFFERY, A. W. H. (1971a), An automated technique for the study of the development of cerebral mechanisms subserving linguistic skill, Proc. Roy. Soc. Med., 64. 919-9)22.

BUFFERY, A. W. H. (1971b), Sex differences in the development of hemispheric asymmetry of function in the human brain, Brain Res., 31, 364-365.

BUFFERY, A. W. H. (1974), Asymmetrical lateralisation of cerebral functions and the effects of unilateral brain surgery in epileptic patients, en Dimond, S. J. \& Beaumont, J. G. (eds.), Hemisphere Function in the Human Brain, Elek Science, Londres.

BUFFERY, A. W. H. (1956), Sex differences in the neuropsychological development of verbal and spatial skills, en Knights. R. \& Bakker, D. J. (eds.). The Neuropsychology of Learning Disorders: Theoretical Approaches. Universidad Park Press. Baltimore.

BUFFERY, A. W. H., y GRAY, J. A. (1972), Sex differences in the development of spatial and linguistic skills, en Ounsted, C. \& Taylor, D. C. (eds.), Gender Differences: Their Ontogeny and Significance, Churchill Livingstone, Edimburgo y Londres.

BURGERMEISTER, B. (1962), Psychological Techniques in neurological Diagnosis, Hoeber, Nuevâ Yôth.

BURT, C. (1909), Experimental tests of general intelligence, Br. J. Psychol., 3, 94-177.

BUTTERS, N., y BRODY, B. A. (1968), The role of the left parietal love in mediation of intraand cross-modal association, Cortex, 4, 328-343.

BUTTERS, N., y CERMAK, L. S. (1074), Some comments on Warrington and Baddeley's report on normal short-term memory in amnesic patients, Neuropsychologia, 12, 283-285.

CHAMBERS; W. R. (1955), Neuropsychological conditions masquerading as psychiatric disorders, Am. J. Psychiat., 112, 387-389.

CHAPMAN, L. F., y WOLFF, H. G. (1959), The cerebral hemispheres and the highest integrative functions of man, Arch, Neurol., 1, 357-424.

CHOMSKY, N. (1957), Syntactic Structures, Mouton, The Hague.

CHOMSKY, N. (1959), Review of Verbal Behaviour by Skinner, B. F., Language, 35, 26-58.

CHRISTENSEN, A. L. (1975), Luria's Neuropsychological Investigation, Spectrum Publications, Inc., Nueva York.

ClARE, A. (1976), Psychiatry in Dissent: Controversial Issues in Thought and Practice, Tavistock Publications Ltd., Londres.

CONKEY, R. C. (1938), Psychological changes associated with head injuries, Arch. Psychol., N. ${ }^{\circ} 232$.

COOPER; I. S.; AMIN, I.; CHANDRA; R., y WALTZ, J. M. (1973), A surgical investigation of clinical physiology of the pulvinar complex in man, J. Neurol. Sci., 18, 89-110.

CORKIN, S. (1965), Tactually-guided maze learning in man: Effects of unilateral cortical excisions and bilateral hippocampal lesions, Neuropsychologia, 3, 339-351.

CRITCHLEY, M. (1953), The Parietal Lobes, Arriold, Londres.

CRONBACH, L. J. (1970), Essentials of Psychological Testing (third ed.), Harper \& Row, Publishers, Nueva York.

DARLEY, F. L. (1975), Treatment of acquired aphasia, en Friedlander, W. J. (ed.), Advances in Neurology, vol. 7. Current Reviews of Highex Nervous System Dysfunction, Raven Press, Publishers, Nueva York.

DARLEY, F. L.; BROWN, J. R., y SWENSON; W. M. (1975), Language changes after neurosurgery for parkinsonism, Brain Lang., 2, 65-69.

DAVISON. L. A. (1974), Introduction, en Reitan, R. M. \& Davison, L. A. (eds.), Clinical Neuropsychology: Current Status and Applications, V. H. Winston \& Sons, Washington, D. C.

DEAN, P. (1976), Effects of inferotemporal lesions on the behavior of monkeys, Psychol. Bull., $83,41-71$.

DE JONG, R. N. (1967), The Neurologic Examination, Hoeber, Nueva York.

DE RENZI, E.; FAGLIONI, P., y SCOTTI, G. (1970), Hemispheric contribution to exploration of space through the visual and tactile modality, Cortex, 6, 191-203.

DEUTSCH, J. A. (1960), The Structural Basis of Behavior, Universidad de Chicago Press, Chicago.

DICARA, L. V.; BARBER, T. X.; KAMIYA, J.; MILLER, N. E.; SHAPIRO, D., y STOYVA, J. (1975), eds., Biofeedback and Self Control 1974, Aldine Publishing Company, Chicago.

DI CHIRO, G. (1962), How rẹliable is neuroradiology. Neurology (Minneap.), 12, 83-98. 
DIKMAN, S.; MATTHEWS, C. G., y HARLEY, J. P. (1975). The effect of early vs late onset of major motor epilepsy upon cognitive intellectual performances, Epilepsia, 16, 73-81.

DIMOND, S. J. (1972), The Double Brain, Churchill Livingstone, Edimburgo y Londres.

DIMOND; S. J., y BEAUMONT; J. G. (1974), Hemisphere Function in the Human Brain, Elek Science, Londres.

DREWE, E. A.; ETTLINGER, G.; MILNER; A. D., y PASSINGHAM, R. E, (1970). A comparative review of the results of neuropsychological research on man and monkey, Cortex, 6 , 129-163.

EYSENCK, H. J. (1976), Behaviour therapy - dogma or applied science, en Feldman, M. P., \& Broadhurst, A. (eds.), Theoretical and Experimental Bases of the Behaviour Therapiers, John Wiley \& Sons Ltd., Londres.

FAIRWEATHER; H. (1974), Choice reaction times in children. Tesis doctoral sin publicar, Universidad de Oxford.

FAIRWEATHER, H. (1976), Sex differences in cognition, Cognition, 4, 231-280.

FEDIO, P., y VAN BUREN, J. M. (1975), Memory and perceptual deficits during electrical stimulation in the ledt and right thalamus and parietal subcortex, Brain Lang., 2, 78-100.

FELDMAN, M. P., y BROADHURST, A. (1976), eds., Theoretical and Experimental Bases of the Behaviour Therapies, John Wiley \& Sons Ltd., Londres.

FITZHUGH, K. B., y FITZHUGH, L. C. (1965), Effects of early and later onset of cerebral dysfunction upon psychological test performance, Percept. Mot. Skills., $2^{\circ}, 1099-1100$.

FLOWERS, K. A. (1972), Amnesia in Cerebral Dysfunction. Tesis doctoral sin publicar, Universidad de Cambridge.

FLOWERS, K. (1975), Ballistic and corrective movements on an aiming task, Intention tremor and parkinsonian movement disorders compared. Neurology (Minneap.), 25, 413.421.

FREIDES, D. (1974), Human information processing and sensory modality: cross-modal functions, information complexity, memory and deficit, Psychol. Bull, 81, 284-310.

FRITSCH, G., y HITZIG, E. (1870), On the electrical excitability of the cerebrum, in von Bonin, G. (ed.), Some Papers on the Cerebral Cortex, Charles C. Thomas, Springfield, Illinois, 1960 (traducción del artículo alemán, 1890).

GARDNER; R. A., y GARDNER, B. T. (1969), Teaching sign language to a chimpanzce, Science, 165, 664-672.

GARNER, W. R. (1962), Uncertainty and Structure as Psychological Concepts, John Wiley \& Sons Inc., Nueva York, Londres.

GAZE, R. M., y KEATING, M. J. (1974) eds., Development and Regeneration in the Nervous System, Brit. Med. Bull., 33, N. ${ }^{\circ} 2$.

GAZZANIGA, M. S. (1970), The Bisected Brain, Appleton-Century-Crofts, Educational Division Meredith Corporation, Nueva York.

GAZZANIGA, M. S.; BOGEN, J. E., y SPERRY, R. W. (1962), Some functional effects of sectioning the cerebral commissures in man, Proc. Natl. Acad. Sci., 48, 1765-1766.

GAZZANIGA, M. S.; BOGEN, J. E., y SPERRY, R. W. (1965), Observations on visual perception after disconnection of the cerebral hemispheres in man, Brain, 88, 221-236.

GESCHWIND, N. (1965), Disconnexion syndromes in animals and man, Brain, 88, 237-294; 585-644.

GESCHWIND, N., y LEVITSKY, W. (1968), Human brain: Left-right asymmetries in temporal speech region, Science, 161, 186-187.

GILBERT, J. (1969), Clinic al Psychological Tests in Psychiatric and Medical Practice, Charles, C. Thomas, Springfield, Illinois.

GOLDSTEIN, K. (1952). The effects of brain damage on he personality, Psychiatri, 15, 245-260.

GRAY, J. A. (1976), The behavioural inhibition system: A possible substrate for anxiety en Feldman, M. P. \& Broadhurst, A. (eds.), Theoretical and Experimental Bases of The Behaviour Therapies, John Wiley \& Sons Lid., Londres.

HALSTEAD; w. C. (1947), Brain and Intelligence: A Quantitative Study of the Frontal Lobes, Universidad de Chicago Press, Chicago.

HALSTEAD, W. C. (1951), Bilogical intelligence, J. Personality, 20, 118-130.

HALSTED, W. C., y RENNICK, P. M. (1966), Perceptual cognitive disorders in children en Kidd, A. H. \& Rivoire, J. L. (eds.), Perceptual Development in Children, International University Press, Nueva York.

HALSTEAD, W. C., y WEPMAN, J. M. (1949), The Halstead-Wepman Aphasia screening test, J. Speech. Hear. Dis., 14, 9-13. 


\section{Estudios}

HARRIS; J. (1965), Depression and hysteria as symptoms of brain tumor, Henry Ford Hospital Med. Bull., 13, 457.459.

HEBB, D. O. (1949), Organization of Behavior: A Neuropsychological Theory, John Wiley \& Sons. Inc., Nueva York.

HECAEN, H., y AJURIAGUERRA, J. (1964), Left Handedness, Manual Superioriry, and Cerebral Dominance (Ponder, E., Trans.), Grune \& Stratton, Nueva York.

HECAEN, H., y ASSAL, G. (1970), A comparison of constructive deficits following right and left hemispheric lesions, Neuropsychologia, 8, 289-303.

HEIMBURGER, R. F., y REITAN, R. M. (1961), Easily administered written test for lateralizing brain lesions, $J$. Neurosurg., 18, 301-312.

HERNANDEZ PEON, R. (1975), Some neurophysiological models in psychopathology, en Keitzman, M. L., Sutton, S. \& Zubin, J. (eds.), Experimental Approaches to Psychopathology, Academic Press, Nueva York.

HERNANDEZ-PEON, R., y BRUST-CARMONA, H. (1961), Functional role of subcortical structures in habituation and conditioning, en Delafresnaye, J. F. (ed.), Brain Mechanisms and Learning, Blackwell, Oxford.

HINES; D., y SATZ, P. (1974), Cross-modal asymmetries in perception related to asymmetri in cerebral function, Neuropsychologia, 12, 239-247

HIRSCH, H. V. B., $;$ JACOBSON, M. (1975), The perfectible braini: Principles of ncuronal development en Gazzaniga, M. S. \& Blakermore, C. (eds.), Handbook of Psychobiology, Academic Press, Nueva York, San Francisco, Londres.

HOBBS, G. E. (1963), Brain tumors simulating psychiatric disease, Can. Med. Assoc. J., 88, 186-188.

HOOK, O. (1969), Comments on the rehabilitation of the brain-injured, en Walker, A. E., Caveness, W. F. \& Critchley, M. (eds.), The Late Effects of Head Injury, Charles C. Thomas, Springfield, Illinois.

HUBEL, D. H., y WEISEL, T. N. (1959), Receptive fields of single neurons in the cat's striate cortex, J. Physiol., 48, 574.591.

HUTT, C. (1972), Neuroendocrinological behavioural and intellectual aspects of sexual differentiation in human development, en Ounsted, C. \& Taylor, D. C. (eds.), Gender Differences: Their Ontogeny and Significance, Churchill Livingstone, Edimburgo y Londres.

ILLICH, I. (1976), Medical Nemesis: The Expropriation of Health, Marian Boyars Publishers Ltd., Londres.

ISAACSON, R. L. (1975), The myth of recovery from early brain damage, en Ellis, N. R. (ed.), Aberrant Development Infancy, Halsted \& Wiley, Nueva York.

IVERSEN, S. D. (1973), Brain lesions and memory in animals, en Deutsch, J. A. (ed.), The Physiological Basis of Memory, Academic Press, Nueva York y Londres.

JACKSON, H. (1874), On the duality of the brain, Med. Press 1, 19. Reimpreso en Selected Writings of John Hughlings Jackson, Taylor, J. (ed.), vol. 2, Hodder \& Stoughton, Londres (1932).

JOHN, E. R., y KLEINMAN, D. (1975), stimulus generalization between differentiated visual, auditory and central stimuli, $J$. Neurophysiol., 38, 1015-1034.

JOYNT, R. J., y GOLDSTEIN, M. N. (1975), Minot cerebral hemisphere, en Friedlander W. J. (ed.), Advances in Neurology, vol. 7, Current Reviews of Higher Nervous System Dysfunction, Raven Press, Publishers, Nueva York.

KESNER, R. (1973), A neural system analysis of memory storage and retrieval Psychol. Bull., $80,177.203$.

KIMURA, D. (1963a), Right temporal lobe damage, Arch. Neurol. (Chicago), 8, 264-271.

KIMURA D. (1863b), A note on cerebral dominance in hearing, Acta-Oto-laryngol., 56, 617-618.

KIMURA, D. (1966), Dual functional asymmetry of the brain in visual perception, Neuropsy. chologia, 4, 275-285.

KIMURA; D. (1967), Functional asymmetry of the brain in dichotic listening, Cortex, 3. 163-178.

KINSBOURNE, M. (1972), Eye and head turning indicates cerebral lateralization, Science, 176, 539-541.

KINSBOURNE, M. (1973), The control of attention by interaction between the cerebral hemispheres in Kornblum, S. (ed.), Attention and Performance, 4, Academic Press, Nueva York y Londres.

KINSBOURNE, M. (1974a), Lateral interactions in the brain, in Kinsbourne, M. \& Lynn Smith, W. (eds.), Hemispheric Disconnection and Cerebral Function, Charles C. Thomas, Publisher, Springfield, Illinois. 
KINSBOURNE, M. (1974b), Mechanisms of hemispheric interaction in man, in Kinsbourne, M. \& Lynn Smith, W. (eds.), Hemispheric Disconnection and Cerebral Function, Charles C. Thomas, Publisher, Springfield, Illinois.

KINSBOURNE, M., y SMITH, W. L. (1974), Hemispheric Disconnection and Cerebral Functicn, Charles C. Thomas, Publisher, Springfield, Illinois.

KLONOFF, H., y LOW, M. (1974), Disordered brain function in young children and early adolescents: Neuropsychological and electroencephalographic correlates, en Reitan, R. M. \& Davison, L. A. (eds.), Clinical Neuropsychology: Current Status and Applications, V. H. Wins. ton \& Sons, Washington, D. C.

KLONOFF, H., y PARIS, R. (1974), Inmmediate, short-term and residual effects of acute head injuries in children: Neuropsychological and neurological correlates, en Reitan, R. M. \& Davison, L. A. (eds.), Clinical Neuropsychology: Current Status and Applications, V. H. Winston \& Sons, Washington. D. C.

KLOVE, H. (1974), Validation studies in adult clinical neuropsychology, en Reitan, R. M. \& Davison, L. A. (eds.), Clinical Neuropsychology: Current Status and Applications, V. H. Winston \& Sons, Washington, D. C.

KONORSKI, $\mathrm{K}$. (1959), A new method of physiological investigation of recent memory in animals Bull. Acd. Pol. Sci., 7, 11.5-117.

LANSDELL, H. (1962), A sex difference in effect of temporal-lobe neurosurgery on design preference, Nature, 194, 852-854.

LANSDELL, H. (1964), Sex differences in hemispheric asymmetries of the human brain, Natu$r e, 203,550$.

LANSDELL, H. (1971), A general intellectual factor affected by temporal lobe dysfunction, J. Clin. Psychol., 27, 182-184.

LASHLEY, K. S. (1929), Brain Mechanisms and Intelligence, University of Chicago Press, Chicago.

LENNEBERG, E. H. (1967), Biological Foundations of Language, John Wiley \& Sons, Inc., Nueva York, Londres, Sydney.

LEVERE, T. E. (1975), Neural stability, sparing, and behavioral recovery following brain damage, Psychol. Rev., 82, 344.358.

LEVITA, E., y RIKLAN, M. (1973), On subcortical correlates of verbal functions in parkinsonism: Critical consideration, J. Gen. Psychol., 89, 3-9.

LINDEN, E. (1976), Apes, Men and Language, Penguin Books Ltd. Harmondsworth, Middlesex, Inglaterra.

LISHMAN, W. A. (1966), Psychiatric disability after head injury, The significance of brain damage, Proc. Roy. Soc. Med., 59, 261-266.

LISHMAN, W. A. (1968), Brain damage in relation to psychiatric disability after head injury, Br. J. Psychiat., 114, 373-410.

LiSHMAN, W. A. (1973), The psychiatric sequelae of head injury, A review, Psychol. Med., 3, 304-318.

LOCKARD, R. B. (1971), Reflections on the fall of comparative psychology: Is there a message for us all, Amer, Psychol., 26, 168-179.

LUKIANOWICZ, M. S. (1974), Age and sex differences in the development and interaction of some verbal and conservation skills. Tesis doctoral sin publicar. Universidad de Cambridge.

LURIA, A. R. (1948), The Restoration of the Brain Function after War Trauma (ruso) U.S.S.R. Academy of Sciences Press, Moscow (inglés. Restoration of Function after Brain Trauma. Pergamon Press, Londres 1963).

LURIA, A. R. (1958), Brain disorders and language analysis, Lang. Speech, 1, 14-34.

LURIA, A. R. (1962), Higher Cortical Functions in Man (ruso) Moscú, (inglés: Basic Books and Plenum Press, Nueva York y Tavistock, Londres 1966).

LURIA, A. R. (1963), Human Brain and Psychological Processes (ruso) Moscú (inglés: Harper \& Row, Nueva York y Londres 1966).

LURIA, A. R. (1967), Neuropsychology and its significance for behavioral science, Psychologia (Intern. J. Psychol. Orient), 10, 1-6.

LURIA, A. R. (1968), The Mind of a Mnemonist, Basic Books, Inc. Nueva York.

LURIA, A. R. (1971), The World Lost and Re-gained (ruso), Moscu, The Man with a Shattered World (inglés) Basic Books, Inc. Nueva York 1972.

LURIA, A. R. (1973), The Working Brain: An Introduction to Neuropsychology, Penguin Books Ltd. Harmondsworth, Middlesex, Inglaterra.

LURIA, A. R.; NAYDIN, V. D.; TSVETKOVA, L. S., y VINARSKAYA, E. N. (1969), Restoration of higher cortical functions foliowing local brain damage, en Vinken, P. J. \& Btuyn, 
G. W. (eds.), Handbook of Clinical Neurology, vol. 4: Disorders of Speech, Perception and Symbolic Behaviour, North Holland Publishing Company, Amsterdam.

MCAFEE, J. G., y TAXDAL, D. R. (1961), Comparison of radioisotope scanning with cerebral angiography and air studies in brain tumor localization, Radiology, 72, 207-222.

MACCOBY, E. E., y JACKLIN, C. N. (1975), The Psychology of Sex Differences, Oxford University Press, Londres.

MALAMUD, N. (1967), Psychiatric disorder with intracranial tumors of limbic system, Arch, Neurol., 17, 113-123.

MARSDEN: C. D. (1975), The neuropharmacology of abnormal involuntary movement disorders (The Dyskinesias), en Williams, D. (ed.), Modern Trends in Neurology, 6, Butterworths, Londres.

MARSDEN, C. D., y PARKES, J. D. (1973), Abnormal movement disorders, Brit, J. Hosp. Med., 10, 428-450.

MARTTILA, I., y HEISKANEN, O. (1970), Value of neurological and angiographic signs as indicators of the ruptured aneurysm in patients with multiple intracranial ancurysms, Acta Neurochir (Wien), 23, 95-102.

MEIER, M. J. (1974), Some challenges for clinical neuropsychology, en Reitan, R. M. \& Davison, L. A. (eds.), Clinical Neuropsychology: Current Status and Applications, V. H. Winston $\&$ Sons. Washington, D. C.

MEYER, V., y YATES, A. J. (1955), Intellectual changes following temporal lobectomy for psychomotor epilepsy, J. Neurol. Neurosurg. Psychiat., 18. 44-52.

MILLER; G. A; GALANTER, E., y PRIBAM, K. H. (1960), Plans and Structure of Bebavior, Holt, Rinchart \& Winston Inc., Londres, Nueva York, Sydney, Toronto.

MILNER, B. (1958), Psychological defects produced by temporal lobe excision. Res. Publ. Ass. Nerv. Ment. Dis., 36, 244-257.

MILNER; B. (1957), The memory defect in bilateral hippocampal lesions, Psychiat. Res. Rep., $11,43-58$.

MILNER; B. (1962), Laterality effects in audition, en Mountcastle, V. B. (ed.), Interhemispheric Relations and Cerebral Dominance, John Hopkins Press, Baltimore.

MILNER, B. (1963), Effects of different brain lesions on card sorting, Arch Neurol (Chicago), 9, $90-100$.

MILNER, B. (1964), Some effects of frontal lobectomy in man, en Warren, J. M. \& Akert, K. (eds.), The Frontal Granular Cortex and Behavior, McGraw-Hill Book Company, Nueva York.

MILNER, B. (1965), Visually-guided maze learning in man: Effects of bilateral hippocampal, bilateral frontal and unilateral cerebral lesions, Neuropsychologia, 3, 317-338.

MILNER; B. (1967), Brain mechanisms suggested by studies of temporal lobes, en Millikan, C. H. \& Darley, F. L. (eds.), Brain Mechanisms Underlying Speech and Language, Grune \& Stratton, Nueva York y Londres.

MILNER, B. (1968a), Disorders of memory after brain lesions in man, Preface: Material-specifie and generalized memory loss, Neuropsychologia, 6, 175-179.

MILNER, B. (1968b), Visual recognition and recall after right temporal-lobre excision in man, Neurops;chologia, 6, 191-209.

MILNER, B. (1970), Memory and the medial temporal regions of the brain, en Pribram, K. H. \& Broadbent, D. E. (eds.), Biology of Memory, Academic Press, Nueva york, Londres.

MILNER, B. (1971), Interhemispheric differences in the localization of psychological processes in man, en Summerfield, A. (ed.), Cognitive Psychology Br. med. Bull., 27, 272-277.

MILNER, B.; CORKIN, S., y TEUBER, H.-L. (1968), Further analysis of the hippocampal amnesie syndrome: 14-year follow-up study of H. M., Neuropsychologia, 6, 215-234.

MILNER, B., y TAYLOR, L. (1972), Right hemisphere superiority in tactile pattern-recognition after cerebral commissurotomy: Evidence for nom-verbal memory, Neuropsychologia, 10, 1-15.

MILNER, B., y TEUBER, H.-L. (1968), Alteration of perception and memory in man: Reflections on methods, en Weiskrantz, L. (ed.), Analysis of Behavior Change, Harper \& Row, Nueva York.

MISHKIN, M., y FORGAYS, D. G. (1952), Word recognition as function of retinal locus, J. Exp. Psychol., 43, 43-48.

MOHR, J. P.; WATTERS, W. C., y DUNCAN, G. W. (1975), Thalamic hemorrhage and aphasia, Brain Lang., 2, 3-17.

MOWRER, O. H. (1960), Learning Theory and Behavior, John Wiley \& Sons, Nueva York, Londres. 
MULDER, D. W. (1959), Psychoses with brain tumors and other chronic neurologic disorders, en Arieti, S. (ed.), American Handbook of Psychiatry, 2, Basic Books, Nueva York.

NEISSER, U. (1967), Cognitive Psychology, Appleton-Ventury-Crofts, Nueva York.

NEWCOMBE, F. (1969), Missile Wounds of the Brain, Oxford Neurological Monographs, Oxford University Press, Oxford.

NIELSEN, J. M. (1946), Agnosia, Apraxia, Aphasia: Their Value in Cerebral Localization (segunda ed.), Hoeber, Nueva York.

OJEMANN, G. A. (1974), Mental arithmetic during human thalamic stimulation, Neuropsychologia, $12,1-10$.

OJEMAN, G. A. (1975a) ed., Brain and Language, 1, 1-2.

OJEMAN, G. A. (1975b), Language and the thalamus: Object naming and recall during and after thalamic stimulation, Brain Lang, 2, 101-120.

OJEMAN, G. A., y FEDIO, P. (1968), Effect of stimulation of the human thalamus and parietal and temporal white matter on short-term memory, J. Neurosurg., 29, 51-59.

OJEMAN, G. A.; FEDIO, P., y VAN BUREN, J. M. (1968), Anomia from pulvinar and subcortical parietal stimulation, Brain, 91, 99-116.

OLDFIELD, R. C. (1971), The assessment and analysis of handedness, The Edinburgh inventory, Neuropsychologia, 9, 97-113.

OLDFIELD, R. C., y WILLIAMS, M. (1961), Cerebral trauma in infancy and intellectual defect, J. Neurol. Neurosurg. Psychiat., 24, 32-36.

OLDS, J., y MILNER, P. (1954), Positive reinforcement produced by electrical stimulation of septal area and other regions of rat brain, J. Comp. Physiol. Psychol., 47, 419-427.

OLIN, H., y WEISMAN, A. (1964), Psychiatric misdiagnosis of early neurological disease, J.A.M.A., 189, 533-538.

PAIVIO, A. (1971), Imagery and Verbal Processes, Holt, Rinehart \& Winston, Inc., Nueva York.

PARKES, J. D., y MARSDEN, C. D. (1973), The treatment of Parkinson's disease, Brit, J. Hosp. Med. 10, 284-294.

PENFIELD, W. (1965), Conditioning the uncommitted cortex for language, Brain, 88, 787-798.

PENFIELD, W., y MILNER; B. (1958), The memory deficit producir by bilateral lesions of the hippocampal zone, Arch.. Neurol. Psychiat., 79, 475.497.

PERRET, E.; EGGENBERGER; E., y SIEGFRIED, J. (1970), Simple and complex finger movement performance of patients with parkinsonism before and after a unilateral stereotaxic thalamotomy, J. Neurol. Neurosurg. Psychiat., 33, 16-21.

PIERCY, M. (1964), The effect of cerebral lesions on intellectual function: A review of current research trends, Brit. J. Psychiat., 110, 310-352.

PINCUS, J. H., y TUCKER, G. (1974), Behavioral Neurology, Oxford University Press, Londres, Toronto, Nueva York.

PREMACK, D. (1971), Language in the chimpanzee, Science, 172, 808-822.

PRIBRAM, H. K. (1954), Toward a science of neuropsychology, en Patton, R. A. (ed.), Current Trends in Psychology and the Behavioral Sciences, University of Pittsburgh Press.

PRIBRAM, K. H. (1971), Language of the Brain: Experimental Paradoxes and Principles in Neuropsychology, Prentice-Hall, Engiewood Cliffs, N. J.

PRIBRAM, K. H., y TUBBS, W. E. (1967), Short term memory, parsing and the Primate frontal cortex, Science, 156, 1765-1767.

RACHMAN, S. J. (1976), Observational learning and therapeutic modelling, en Feldman, M. P. \& Broadhurst. A. (eds.), Theoretical and Experimental Bases of The Behaviour Therapies, John Wiley \& Sons Ltd., Londres.

RACHMAN, S. J., y PHILIPS, C. (1975), Psychology and Medicine, Maurice Temple Smith Ltd., Londres.

RAMIER, A.-M., y HECAEN, H. (1970), Rôle respectif des atteintes frontales et de la latéralisation lésionnelle dans les déficits de la «fluence verbale», Revue neurol., 123, 17-22.

RASMUSSEN, P.; BUHL, J.; BUSCH, J.; HAASE, J., y HARMSEN, A. (1970), Brainscanning cerebral seintiography: An evaluation of the diagnostic value of scanning compared to other methods of investigation based on 981 patients, Acta Neurochir. (Wien), 23, 103-109.

REITAN, R. M. (1955), An investigation of the validity of Halstead's measures of biological intelligence, Arch. Neurol. Psychiat., 73, 28-35.

REITAN, R. M. (1956), Investigation of relationships between «psychometric and «biological» intelligence, J. Nerv. Ment. Dis., 123, 536-541.

REITAN, R. M. (1959), The comparative effects of brain damage on the Halstead Impairment Indez and the Wechsler-Bellvue Scale, J. Clin. Psychol., 15, 281-285. 


\section{Estudios}

REITAN, R. M. (1962), Psychological Deficit, en Farnsworth. P. R. McNemar, O. \& McNemar, Q. (eds.). Annual Review of Psychology, 13. 415-444. Annual Reviews Inc. Palo Alto, California.

REITAN, R. M. (1964). Psychological deficits resulting from cerebral lesions in man, en Warren, J. M. \& Akert. K. A. (eds.). The Frontal Granual Cortex and Behavior. McGraw-Hill, Nueva York.

REITAN, R. M. (1966a), Problems and prospects in studying the psychological correlates of brain lesions, Cortez, 2, 127-154.

REITAN. R. M. (1966b), A research program on the psychological effects of brain lesions in human beings, en Ellis, N. R. (ed.), International Review of Research in Mental Retardation. 1 . en Academic Press, Nueva York.

REITAN, R. M. (1969), Manual for Administration of Neuropsychological Test Batteries for Adults and $C$ hildren. Publicación privada del autor, Indianapolis.

REITAN, R. M. (1974), Methodological problems in clinical neuropsychology, en Reitan, R. M. \& Davison, L. A. (eds.), Clinical Neuropsychology: Current Status and Applications. V. H. Winston \& Sons, Washington, D. C.

REITAN, R. M. (1976), Neurological and physiological bases of psychopathology, en Rosenzweig, M. R. \& Porter, L. W. (eds.), Annual Review of Psychology, 27, Annual Reviews Inc. Paío Álto, Cailifornia.

REITAN. R. M., y DAVISON, L. A. (1974) eds., Clinical Neuropsychology: Current Status and Applications. V. H. Winston \& Sons, Washington, D. C.

REMINGTON, F. B., y RUBERT, S. L. (1962), Why patients with brain tumor came to a psychiatric hospital: A third year survey, Am. J. Psychiat., 119, 256-257.

REYNELL, W. R. (1943), Discussion on disorders of personality after head injury, Proc. Roy. Soc, Med., 37, 561-564.

REYNELL, W. R. (1944), A psychometric method of determining intellectual loss following head injury, J. Ment. Sci., 90, 710-719.

RICHARDSON, F. (1963), Some effects of severe head injury, Dev. Med. Child Neurol., 5 , $471-482$

RIESE, W. (1959), A History of Neurology, MD Publications, Nueva York.

RIKLAN, M., y COOPER, I. S. (1975), Psychometric studies of verbal functions following thalamic lesions in humans, Broin Long., 2, 45-64.

ROSADINI, G., y ROSSI, G. F. (1967), On the suggested cerebral dominance for consciousness, Brain, 90, $101-112$.

RUSSELL, E. W.; NEURINGER; C., y GOLDSTEIN, G. (1970), Assessment of Brain Damage: A Neuropsychological key Approach, Wiley-Interscience, Nueva York.

RYLANDER, G. (1939), Personality Changes after Operation on the Frontal Lobes: A Clinical Study of 32 Cases. Munksgaard, Copenhage.

RYLE, G. (1949), The Concept of Mind. Hutchinson, Londres.

SATZ, P.; ACHENBACK, K., y FENNELL, E. (1967), Correlations between assessed manual laterality and predicted speech laterality in a normal population, Neuropsychologia. 5. 295-310.

SCHALTENBRAND, G. (1975), The effects on speech and language of stereotactical stimulation in thalamus and corpus callosum. Brain Lang., 2, 70-77.

SCOVILLE; W. B., y MILNER, B. (1957), Loss of recent memory after bilateral hippocampal lesions, J. Neurol. Neurosurg. Psychiat., 20, 11-21.

SELTZER, M. D., y BENSON, D. F. (1974), The temporal pattern of retrograde amnesia in Korsakoff's disease, Neurology (Minneap.), 24, 527-530.

SEMMES, J.; WEINSTEIN, S.; GHENT, L., y TEUBER, H.-L. (1955), Spatial orientation in man after cerebral injury: 1. Analyses by locus of lesion, $J$. Psycbol., 39. 227-244

SHALLICE; T., y WARRINGTON, E. K. (1970), Independent functioning of verbal memory stores: A Neuropsychological study, Quart. J. Exp. Psychol., 22, 261-273.

SHANKWEILLER; D. (1966), Effects of temporal lobe damage on perception of dichotically presented melodies, J. Comp. Physiol. Psychol., 62, $115-119$

SMALL, L. (1973), Neuropsychodiagnosis in Psychotherapy, Bruner/Mazel, Nueva York.

SMITH, A. (1975), Neuropsychological testing in neurological disorders, en Friedlander, W. J. (ed.), Advances in Neurology, vol. 7. Current Reviews of Higher Nervous System Dysfunction. Raven Press, Nueva York.

SMITH, W. L., y PHILIPPUS, M. J. (1969), Neuropsychological Testing in Organic Brain Dysfunction, Charles C. Thomas, Springfield, Illinois. 
SOKOLOV, E. N. (1960), Neuronal models and the orienting influence, en Brazier, M. A. B. (ed.), Central Nervous System and Behavior, Josiah Macey Jr. Foundation, Nueva York.

SPERRY, R. W. (1971), How a developing brain gets itself wired for adaptive function, en Tobach. E., Aronson, L. A. \& Shaw, E. (eds.). The Biopsychology of Development. Academic Press, Nueva York.

SPERRY; R. W.; GAZZANIGA, M. S. y BOGAN, J. E. (1969), Interhemispheric relationships; The neocortical commissures: syndromes of hemisphere disconnection, en Vinken, $P$. J. \& Bruyn, G. W. (eds.), Handbook of Clinical Neurology, vol. 4: Disorders of speech, perception and symbolic behaviour. North Holland Publishing Company, Amsterdam.

SPREEN, O., y BENTON, A. L. (1965), Comparative studies of some psychological tests for cerebral damage. J. Nerv. Ment. Dis., 140, 323-333.

STEIN, D. G.; ROSEN, J. J., y BUTTERS, N. (1974) eds., Plasticity and Recovery of Function in the Central Nervous System, Academic Press, Inc., Nueva York, San Francisco, Londres.

STEPIEN, L., y SIERPINSKI, S. (1960), The effect of focal lesions ot he brain upon auditory and visual recent memory in man, $J$. Neurol. Neurosurg. Psycbiat., 23, 334-340.

STRAUSS. I., y SAVITSKY, N. (1934), Head injury: Neurologic and Psychiatric aspects, Arch. Neurol. Psychiat., 31, 893-955.

TAYLOR, D. C. (1969), Differential rates of cerebral maturation between sexes and between hemispheres, Lancet, ii, 140-142

TEUBER, H. L. (1955), Physiological Psychology, en Stone, C. P. \& McNemar, Q. (eds.), Annual Review of Psychology. 6. Annual Reviews Inc., Aplo Alto, California.

TEVZADZE, V. G. (1974), Formation of conditional reflex by direct electrical stimulation of various brain structures, Zhurnal vysshey nervnoy deyatl nosti imeni 1. P. Pavlova, 1974 , 24.(2): 287-291. En Soviet Neurology and Psychiatry, 8(3) 1975 (Fall) 94-101.

TREVARTHEN, C. B. (1973) Behavioral embryology, en Carterette, E. C. \& Friedman, M. P. (eds.). Handbook of Perception, vol. 3. Biology of Perceptual Systems, Academic Press, Nueva York y Londres.

UMILTA, C.; SNYDER, C., y SNYDER, M. (1972), Repetition effect as a function of eventu uncertainty, response-stimulus interval, and rank order of the event, $J$. Expt. Psychol, 93. $320 \cdot 326$.

VAN BUREN. J. M. (1975), Question of thalamic participation in speech mechanisms Brain Lang. . 2. $31-44$.

VILKKI, J., y LAITINEN, L. V. (1974), Differential effects of left and right ventrolateral thalamotomy on receptive and expressive verbal performances and face-matehing. Neuropsycbologia. 12, 11-19.

VINKEN, P. J.. y BRUYN, G. W. (1969a) eds., Handbook of Clinical Neurology, vol. 3: Disorders of Higher Nervous Activity. North Holland Publishing Company, Amsterdam.

VINKEN, P. J., y BRUYN, G. W. (1969b) eds., Handbook of Clinical Neurology, vol. 4: Disorders of Speech. Perception and Symbolic Behaviour. North Holland Publishing Company, Amsterdam.

VON MONAKOW, C. (1911), Lokalization der Hirnfunktionen, J. Psychol. Neurol., 17, $185,200$.

VON MONAKOW, C. (1914), Die Lokalization im Frosshirn und der Abbau der Funktion Durch Kortikale Herde. Bergmann, Wiesbaden.

WADA. J. A.. y RASMUSSEN, T. (1960). Intracarotid injection of sodiut, amytal for the lateralization of cerebral speech dominance. Experimental and clinical observations, J. Neurosurg., 17. $266-282$.

W AGGONER, R. W. y BAGCHI, B. K. (1954), Initial masking of organic brain changes by psychiatric symptoms: Clinical and EEG studies, Am.J. Psychiat., 110, 904-910.

WALKER, A. E.; CAVENESS, W. F., y CRITCHLEY. M. (1966) eds., The Late Effects of Head Injury. Charles C. Thomas, Springfield, Illinois.

WALTON. J. N. (1975), Essentials of Neurology (fourth ed.), Pitman Medical Publishing Co. Ltd. Londres.

W ARRINGTON. E. K. (1969), Constructional apraxia, en Vinken, P. J. \& Bruyn, G. W. (eds.), Handbook of Clinical Neurology, vol. 4: Disorders of Speech. Perception and Symbolic Beha. niour. North Holland Publishing Company, Amsterdam.

WARRINGTON, E. K. (1971). Neurological disorders of memory, en Summerfield, A. (ed.). Cognitive Psychology, Br. med. Bull., 27, 243-247.

WARRINGTON, E. K. y JAMES, M. (1967), An experimental investigation of facial recognition in patients with unilatera! cerebra! lesions, Cortex, 3, 317-326. 


\section{Estudios}

WARRINGTON: e. K.. y PRATT, R. T. (1973), Language laterality in left-handers assessed by unilateral E.C.T., Neuropsychologia, 11, 423-428.

WARRINGTON, E. K. y SHALLICE; T. (1969), The selective impairment of auditory verbal short-term memory, Brain, 92, 885-896.

WARRINGTON; E. K., y WEISKRANTZ, L. (1973), An analysis of short-term and long-term memory defects in man, en Deutsch, J. A. (ed.), The Physiological Basis of Memory, Academic Press, Nueva York y Londres.

WECHSLER, I. S. (1963). Clinical Neurology, Straus, Filadelfia.

WHITE, S. R. (1975), Mnemonic strategies in normal and head-injured children. Tesis doctoral sin publicar, Universidad de Oxford.

WHITTY, C. W. M. (1956), Mental changes as a presenting feature in subcortical cerebral lesions, J. Ment. Sci, 102, 719-725.

WITELSON, S. (1974). Hemispheric specialization for linguistic and non-linguistic tactual perception using a dichotomous stimulation technique, Cortex, 10, 3-17.

WITELSON, S. F., y PALLIE; W. (1973), Left hemisphere specialization of language in the newborn: Neuroanatomical evidence of asymmetry, Brain, 96, 641-646.

WOLPE, J. (1958), Psychotherapy by Reciprocal Inbibition, Stanford University Press.

WYKE, M. (1971), Dysphasia, en Summerfield, A. (ed.), Cognitive Psychology, Br. med. Bull., $27,21 ! 2 ! 7$

YATES, A. J. (1954). The validity of some psychological tests of brain damage, Psychol. Bull., 51.359-379.

YENI-KOMSHIAN, G. H., y BENSON, D. A. (1976), A natomical study of cerebral asymmetry in the temporal lobe of humans, chimpanzees, and rhesus monkeys, Science, 192, 387-389.

YNTEMA; D. B., y TRASK, F. B. (1963), Recall as a search process, J. verb. Learn. Verb. Behav., 2, 65-74

YOUNG, R. M. (1970), Minf. Brain and Adaptation in the Nineteenth Century, Clarendon Press, Oxford.

ZANGWILL, O. L. (1960), Cerebral Dominance and its Relation to Psychological Function Oliver \& Boyd, Edimburgo.

ZANGWILL, O. L. ' 1962), Handedness and dominance, en Money, J. (ed.), Reading Disabilities, John Hopkins Press, Baltimore, Maryland.

ZANGWILL, O. L. (1966), Psychological deficits associated with frontal lobe lesion, Int. J. Neuroi., $5,395-402$.

ZANGWILL, O. L. (1967), Speech and the minor hemisphere, Acta Neurol. Psychiat. Belg., 67, 1013-1020. 